\title{
Perspectives and Approaches of Primary Care Physicians on Diagnosing Dementia- a Qualitative Japan - United States Comparison Study
}

\section{Michiko Abe}

Hamamatsu University School of Medicine

\section{Shinji Tsunawaki}

Omaezaki Family Medicine Center

\section{Melissa DeJonckheere \\ University of Michigan-Ann Arbor}

Christine T. Cigolle

University of Michigan-Ann Arbor

Kristin Phillips

VA Ann Arbor Healthcare System

\section{Ellen B. Rubinstein}

North Dakota State University

\section{Masakazu Matsuda}

Shizuoka Family Medicine Program

Michael D. Fetters ( $D$ mfetters@med.umich.edu )

University of Michigan-Ann Arbor

\section{Machiko Inoue}

Hamamatsu University School of Medicine

\section{Research Article}

Keywords: diagnosing dementia, primary care physicians, Japan, United States, qualitative comparison

Posted Date: February 16th, 2021

DOl: https://doi.org/10.21203/rs.3.rs-198779/v1

License: (c) (i) This work is licensed under a Creative Commons Attribution 4.0 International License.

Read Full License 


\section{Abstract}

Background: To explore the perspectives and approaches of primary care physicians in Japan and the US on diagnosing dementia.

Methods: Qualitative comparison conducted in ethnographic tradition using semi-structured interviews and thematic analysis. Primary care settings across Japan and in the Midwest State of Michigan, US. Participants were a total of 48 primary care physicians, 24 each from Japan and the US participated. Both groups contained a mixture of the practice area (rural/urban), gender, age, and years of experience as a primary care physician.

Results: Participants in Japan and the US voiced similar approaches for making the diagnosis of dementia and held similar views about the desire benefits of diagnosing dementia. Differences were found in attitudes about the appropriate timing of formally diagnosing dementia. Japanese physicians tended to make a formal diagnosis when problems that would benefit from long-term care services emerged for family members. US physicians were more proactive in diagnosing dementia in the early stages by screening for dementia in health check-ups and promoting advance directives when the patients were still capable of decision-making. Views about appropriate timing of diagnostic testing for dementia in the two systems reflect what medical or nursing care services physicians can use to support dementia patients and caregivers.

Conclusions: Testing to establish an early diagnosis of dementia by primary care physicians only partly relates to testing options available. Benefits of making the diagnosis included the need to activate the long-term care services in Japan and for early intervention and authoring advance directives in the US.

\section{Background}

In 2015 , about 47 million people were living with dementia globally and the number is projected to triple by 2050 [1]. Given the sheer volume of patients with dementia, primary care physicians have an increasingly important role in diagnosing and managing dementia in their daily practice [2-4]. While clear evidence for clinical utility of screening for dementia in primary care setting remains limited [5-7], many authors have suggested potential benefits of early diagnosis by arguing that therapeutic intervention could delay progression of dementia $[1,3,8,9]$, mobilize support that could help maintain quality of life for patients and their family members [3, 4, 10-12], and provide opportunity to discuss advance care planning or advance directives with dementia patients [10,13-15].

However, there are many barriers in primary care settings to establishing the diagnosis of dementia early. Barriers noted include limited time for consultation while managing multiple chronic conditions $[2,3,12]$, uncertainty about diagnostic resources including concerns about making diagnostic errors [3, 16], and limited effectiveness and the high cost of treatment $[12,16]$. Additionally, concerns have included increasing the fear or stigma of patients and family members [2, 16], and lacking resources in the community for supporting dementia patients and family members $[16,17]$. Weighing the benefits of an 
early diagnosis of dementia is a complex arena for primary care physicians trying to pursue a 'best interests' approach for patients and their care providers [16].

Previous research about diagnosing dementia in the primary care setting has largely been conducted in the United States (US), United Kingdom, and Australia [6]. Even though Japan is one of the most aged countries in the world and the social burden of dementia is growing $[18,19]$, few studies have described the perspectives of Japanese primary care physicians on this issue. Previous scholarship has noted that dementia has different sociocultural contexts [20-22] and medical practice is affected by public health measures and local care resources [23-25]. Therefore, understanding facilitators or barriers for establishing a dementia diagnosis unique to Japanese primary care physicians [26] could be further contextualized by comparing practices in Japan with practices in different setting [27].

The premise for this research is that cross national research on establishing a diagnosis of dementia could enhance understanding Japanese and US primary care physicians' approaches to patient care. To contextualize this comparison, several factors should be considered. First, both countries are experiencing rapid aging in their populations. The share of the population over the age of 65 is $27.7 \%$ in Japan and $15.6 \%$ in the US [28-30]. Both countries have a large population of patients with dementia. There are an estimated 6.3 million persons in Japan and 5.8 million in the US with dementia even though the latter has more than twice the total population [31-33].

Second, the elderly in both countries have access to health insurance but with some important differences. Japan has offered universal health care coverage since 1961 [34]. Long-term care insurance was established in 2000 as a component of national social security to help the elderly with needs associated with accessing health care services $[24,32,35]$. In the US, there have been two primary types of public health insurance systems since 1965: Medicare for citizens of 65 years and older, and Medicaid for low-income citizens and other meeting certain disease criteria. Insurance coverage varies by individual depending on their past and present employment condition [36], and unlike Japan's universal access, long-term care insurance in the US is very expensive and available through private insurers [37]. These differences aside, both countries have technophilic cultures with technology relatively easy to access. Likewise, both health insurance systems incentivize using health care technology [25].

Third, the role and characteristics of primary care physicians differ in the two countries. In Japan's system, patients are allowed to visit any physician or multiple physicians without referral [38]. In contrast, patients typically require referral from their primary care doctors in the US system as an approach to help minimize health care costs [39]. In Japan, a community-based integrated care system was implemented by the government in 2012 to activate seamless healthcare services with a multidisciplinary approach for the elderly and people living with dementia $[31,40]$. Primary care physicians play a central role in this plan, however a formally recognized system of family medicine is still new in Japan. Despite a much longer history in a handful of programs to launch family medicine, systematic residency training programs to produce family physicians didn't start growing until around 2007 [38, 41]. It wasn't until 2017 that sogoushinryou, translated as general medicine, became the 19th specialty in Japan [38]. 
In Japan, the vast majority of physicians who were working as primary care physicians before establishment of the specialty in 2017 had trained first in hospitals as subspecialists before opening a private outpatient practice as a second career [42]. Arguably, they are hybrid subspecialist/primary care physicians based on their provision of a mix of subspecialty care learned during their hospital training, and first contact care for common acute and chronic conditions [43]. Like Japan, family physicians play an important role in communities as the most common primary care provider in the US. In contrast to Japan, family medicine in the US was recognized as a specialty nearly five decades earlier in 1969. In the US, primary care physicians are systematically trained to provide a broad range of care which prepares them to care for a wide range of health issues of patients of various ages in a continuous relationship $[39,42]$. Due to a different system that encourages privileging for local hospital inpatient work, many family doctors, especially those in rural areas, practice a combination of inpatient care in community hospitals and local outpatient care, a source of continuity for hospitalized elderly.

Fourth, the two countries have very different patient and family participation in decision making and sharing of information [25,44]. The US has implemented advance directives since the 1990s [45]. While perspectives on the effectiveness of this approach vary $[13,46]$, advance directives do help formalize surrogate medical decision making when an individual can no longer make decisions. In Japan, the concept of "advance care planning" was introduced in the late 2000s but uptake by physicians is still low [47]. In contrast to the US, the system of advanced care planning does not have legal protection for surrogate medical decision making in Japan [48].

Given these pertinent demographic and sociocultural similarities and differences, the purpose of this study was to explore the perspectives of primary care physicians on diagnosing dementia in Japan and the US. By comparing the similarities and differences of primary care physicians in the US and Japan, as well as between urban and rural areas, we aimed to understand the approach of primary care physicians' for diagnosing dementia.

\section{Methods}

\section{Design}

The present study utilized qualitative methods grounded in an ethnographic tradition that is needed due to the lack of descriptive research about primary care physicians' management of dementia. It is a part of a larger investigation of a rural-urban comparison of primary care physicians' dementia care for multimorbid older adults conducted in Japan and the US. Data collection by semi-structured interviews was done in Japan from August to October in 2017, and in the US between August 2015 and June 2018.

\section{Setting}

In Japan, participants were recruited from physicians residing throughout the country for a national sample. In the US, participants were recruited from primary care physicians in the State of Michigan. Located in the Midwest, this single state has a broad range of industries, agriculture, racial and ethnic 
populations, as well as rural, suburban, and urban settings. Michigan has relatively comparable aging and dementia rates with the US overall and is relatively comparable with Japan as shown in Table 1.

Table 1

Population and dementia prevalence in Japan, US, and Michigan. (2020)

\begin{tabular}{|llll|}
\hline & Japan & U.S. & Michigan \\
\hline Total population & 126 million & 327 million & 10 million \\
\hline Share of the population aged over 65 and older & $27.7 \%$ & $15.6 \%$ & $17.7 \%$ \\
\hline Population living with dementia, 65 and older & 6.3 million & 5.8 million & 0.2 million \\
\hline Dementia population rate among 65 and older & $17.5 \%$ & $11.4 \%$ & $10.7 \%$ \\
\hline $\begin{array}{l}\text { Sources: OECD, 2020; OECD, 2019; United States Census Bureau, 2019; Ministry of Health Labour and } \\
\text { Welfare, 2017; Alzheimer's Association, 2020. }\end{array}$ & & & \\
\hline
\end{tabular}

\section{Data collection instrument and reflexivity}

Based on a review of the literature, and their extensive knowledge of the two country's primary care systems, the team members created the interview guide. We used a semi-structured interview format which allowed the interviewer to ask additional questions of interest as they emerged [49].

Interview questions addressed primary care physicians' goals in managing multimorbid patients with dementia; their practices of diagnosing, disclosing, and managing dementia; their comfort level with these tasks; and available resources for dementia care in their working environment (Supplement 1). We collected trajectories of several memorable cases from each participant and probed about their perspectives and approaches of diagnosing dementia. The investigators developed the interview guide first in English, and then translated it for use in Japan. A professional service translated the interview guide into Japanese and two bilingual senior investigators (MI, MF) reviewed the language and content to ensure the translation wording was natural and appropriate for the Japanese context. The analysis for this study was focused on questions and responses regarding making dementia diagnosis.

Four team members were assigned as interviewers which included a qualitative research methodologist with training in intercultural communication (MA) in Japan, and in the US, a geriatric pharmacist (KP), an anthropologist (ER), and a medical and public health student. All interviewers were trained to conduct qualitative interviews and had no relation with the participants in advance.

\section{Recruitment, sampling and data collection procedures}

The goal of our purposeful sampling was to recruit in both countries a mixture of physicians based on rural/suburban/urban practice location, gender, age, and years of experience as a primary care physician in accordance with maximum variation sampling. In Japan, participants in the research were members of 
Japan Primary Care Association and who had received training as a family physician. We began by recruiting board certified family physician within the host program, who then introduced the interviewer to others who would potentially meet criteria. Because cultural norms generally require an introduction from a known colleague, the enrolled subjects provided contact information for others who meet the maximum variation sampling intent. Specifically, we sought representation from geographical areas in the north and south regions of the country including remote islands, and a broad age range of the primary care physicians. In the US, recruitment began by approaching primary care physicians including family physicians and internists practicing within the Great Lakes Research Into Practice Network (GRIN) [50].

Information regarding the study was distributed to physicians in the network, and those with an interest in participating were contacted to confirm eligibility. To expand access to practitioners' experiences and clinical backgrounds, we expanded our sampling to include primary care physicians from rural and metropolitan areas outside the GRIN network.

Interviews were conducted in person at physicians' offices, telephone/voice over the internet, or using video conferencing. Interviews lasted 60 to 150 minutes in Japan and 30 to 90 minutes in the US. All were audio-recorded and transcribed verbatim. Although the dementia care process was discussed broadly during the interviews in both locations, this paper focuses on primary care physicians' practice and perspectives of diagnosing dementia.

\section{Ethical considerations}

We informed participants about the voluntary nature of study participation and the freedom to withdraw from the study. Human subjects review and ethical approval was obtained from the Institutional Review Board of Hamamatsu University School of Medicine in Japan (No.16-233) and Health Sciences and Behavioral Sciences Institutional Review Board of the University of Michigan in the US (IRB 00000246).

\section{Qualitative data analysis}

After each interview, the interviewer created field notes, using the "3C's approach" [51] addressing the three constructs of "context", "content" and "concepts" to capture the major issues that were discussed during the interview. The Japan team members translated their 3C's summaries into English to promote exchange of the results with the US team several times over the course of data collection. Each team proceeded with a thematic analysis to the transcribed interview data in their language which facilitated an understanding of the perspectives of physicians in their own words. MAXQDA Analytic pro 12 was used for data coding.

For the Japanese data analysis, the research team (MI, ST, MM, MA) regularly shared the 3C's summaries in scheduled research meetings to discuss the key conceptual ideas from each interview. To allow for a naturalistic process not encumbered by language and cultural differences based on the US format, the initial coding scheme in Japan created by the analyst (MA) contained the study's major constructs, e.g., diagnosis, disclosure, management, the end-of-life care, and sub-codes were added in response to emerging findings from the team discussions. For the US data, after the first eight interviews were conducted, the research team began developing a coding scheme. Then three researchers (CC, KP, MD) 
applied the codes to the first eight transcripts and modified the coding scheme by adding additional codes that were not yet reflected. The remaining transcripts were coded by two analysts (MD, ER) and discussed by all team members. After conducting 24 interviews by each team, the research team members agreed that thematic saturation had been reached in both samples. The 3C's summaries were reviewed during data analysis.

To conduct member checking, a process of sharing the overall findings with participants [52], we distributed a summary of the project findings, a copy of each participants' interview summary, and a request for feedback. In Japan, all 24 participants replied with an agreement with the distributed summary, and 22 participants provided additional comments that we incorporated into the findings. In the US, the 24 participants were emailed information about the study findings though one email did not function. The 9 responding participants all indicated agreement and added a few clarifications that we incorporated into the findings.

\section{Japan-US Comparisons}

The lead analyst from Japan (MA) visited the US to compare the data coding and analysis approach with the US team members (MF, MD, CC, KP). This ensured that the coding schemes equivalently functioned for examining constructs of mutual interest. Later in the process, the lead US analyst (MD) visited Japan for a focused discussion of the present study. We first compared the coding scheme between Japan and the US under the category of "diagnosis" and related themes. Because the US had a more detailed scheme, MA reviewed Japan data and conducted additional coding while referencing the US scheme. There were several codes that emerged only from the Japan or the US data. MA and MD discussed the differences and set up several comparable themes such as primary care physicians' approaches to diagnosing dementia, cooperation with specialist physicians, perceived benefits of dementia diagnosis in primary care, and appropriate timing of diagnosing dementia in the views of primary care physicians. For each of these themes, MA first drafted a descriptive summary from Japan data and selected several quotations that represent the findings while MD did the same with the US data. Finally, we compared both descriptive summaries and discussed the similarities and differences and factors that affect the approaches of diagnosing dementia for the participating primary care physicians. The main codes and sub-codes related to "dementia diagnosis" are presented in Table 2. 
Table 2

Coding scheme of perspectives of primary care physicians on diagnosing dementia (2017)

\begin{tabular}{|c|c|}
\hline Main Codes & Sub-codes (*Japan only code, tUS only code) \\
\hline \multicolumn{2}{|l|}{ Timing of diagnosis } \\
\hline \multirow[t]{2}{*}{ Confidence in diagnosis } & High confidence \\
\hline & Low confidence \\
\hline \multirow[t]{11}{*}{ Diagnostic process } & Annual exams \\
\hline & MD observations/knowledge \\
\hline & Patient self-reports symptoms \\
\hline & Family reports symptoms \\
\hline & Clinical staffs/neighbors report symptoms * \\
\hline & Understanding the patient's life history/living environment * \\
\hline & Taking clinical history from family/patient $\dagger$ \\
\hline & Seeing only advanced cases \\
\hline & Diagnostic ambiguity \\
\hline & Assessments \& tests \\
\hline & Collective decision-making with family * \\
\hline Confirming diagnosis & For better diagnostic tools \\
\hline \multirow[t]{7}{*}{ through specialist } & For specialist resources \\
\hline & Because diagnosis is unclear \\
\hline & For acceptance of family/patient \\
\hline & To plan treatment/help with treatment \\
\hline & Lack of access to specialists \\
\hline & Lack of access to neurocognitive testing \\
\hline & Because it's too hard for primary care physician to handle \\
\hline \multirow[t]{5}{*}{ Benefits of diagnosis } & Safety \\
\hline & Planning for the future \\
\hline & Long-term insurance \\
\hline & Access to care resources \\
\hline & Knowing/acceptance of diagnosis by patient/family \\
\hline
\end{tabular}




\section{Main Codes}

Sub-codes (*Japan only code, tUS only code)

Acceptance/stigma in the community

Treatment planning

\section{Results}

A total of 48 primary care physicians, 24 each from Japan and the US participated in the interviews and their profiles are illustrated in Table 3. In Japan, physicians based in local hospitals that often have active outpatient departments were included because they were actively providing primary care services. Five participants had experiences of working in both urban and rural areas. In these cases, we asked them to speak primarily about their experiences in the environment leaving the strongest impression on them and to compare the two environments whenever appropriate. In the US, all physicians (100\%) practiced in clinics. Because Japan is much more densely populated than the US, we used participants' own categorization of their practice setting as suburban versus rural.

Table 3

Characteristics of participants $(n=48)$

\begin{tabular}{|lllll|}
\hline & \multicolumn{3}{l}{ Japan $(\mathbf{n}=\mathbf{2 4 )}$} & US $(\mathbf{n = 2 4 )}$ \\
\cline { 2 - 5 } & Rural & Urban & Rural & Urban \\
\cline { 2 - 5 } & $\mathbf{( n = 1 2 )}$ & $(\mathbf{n = 1 2 )}$ & $\mathbf{( n = 1 2 )}$ & $\mathbf{( n = 1 2 )}$ \\
\hline Years practicing & $12.7 \pm 5.9$ & $17.4 \pm 8.1$ & $7.9 \pm 6.6$ & $19.3 \pm 9.0$ \\
as physician * & $(6-24)$ & $(9-38)$ & $(2-23)$ & $(6-34)$ \\
\hline Gender* & & & & \\
\hline Male & $9(75 \%)$ & $8(67 \%)$ & $5(42 \%)$ & $5(42 \%)$ \\
\hline Female & $3(25 \%)$ & $4(33 \%)$ & $7(58 \%)$ & $7(58 \%)$ \\
\hline Setting** & & & & \\
\hline Clinics & $8(67 \%)$ & $10(83 \%)$ & $12(100 \%)$ & $12(100 \%)$ \\
\hline Hospital & $4(33 \%)$ & $2(17 \%)$ & none & none \\
\hline *Average \pm SD, **numbers(\%) & & & \\
\hline
\end{tabular}

\section{Approaches to diagnosing dementia}

We found that primary care physicians in Japan and the US used a similar approach to diagnosing dementia. Physicians commonly administered a screening test that most typically among these 
participants was the Mini-Mental State Examination (MMSE). They reported conducting blood work to rule out treatable diseases, checking medications to ensure impaired cognition was not a medical side effect, examining for underlying depression or mental illness, and considering use of a brain MRI or CT scan. They reported referring patients occasionally to a non-primary care specialist doctor for more testing to determine a specific type of dementia. In addition to above actions, these physicians emphasized speaking with patients' family members or caregivers to take additional history and to understand the patients' conditions at home. Many felt this information was equally or often times more important than doing other tests for diagnosing dementia.

There were minor differences in the screening tools used in Japan and the US. While the Mini-Mental State Examination (MMSE) was most common in both countries, participants in Japan also reported using the Hasegawa's Dementia Scale (HSD-R) while those in the US used the Montreal Cognitive Assessment (MoCA) and Clock-Drawing test as alternatives.

Attitudes regarding specialist referral varied by the individual. Some participants reported always sending patients to a specialist for a formal diagnosis of dementia while others reported basically making the final diagnosis by themselves except in cases where they felt the need for more detailed testing. Additionally, participants in both countries were influenced whether or not to refer to a dementia specialist according to the wishes of patients' family members.

I may ask for a specialist's advice if there are some concerns. And if the patient's family members are hoping to see a specialist then I will introduce them. Other than that basically, I keep taking care of them. (Japan_Urban_24)

I would say if the Montreal Cognitive Assessment (MoCA) Test is really abnormal, and I feel confident, based on the symptoms, and the caregiver, and all that stuff, I'm going to diagnose. With or without imaging, depending. (US_Urban_M04)

We asked participants about their confidence in making the diagnosis of dementia. Most of them were confident about their knowledge of the procedure for making the diagnosis given their access to specialists in circumstances if they were not comfortable with the case. The level of confidence for making a dementia diagnosis was markedly higher for obvious cases of dementia, for patients with advanced age and cognitive symptoms, and especially when family members could provide a compelling history for the patient. In contrast, situations that lowered the physicians' confidence in making the diagnosis included an early stage of dementia, suspicion of dementia in younger patients, lacking access to family members to discuss the situation or when patients lived by themselves, and a clinical setting which did not have good access to specialists or imaging tests. Varying levels of confidence were consistent between physicians in Japan and the U.S.

The lack of access to specialists was noted particularly in rural areas in both Japan and the US but for different reasons. In Japan, particularly in remote islands, concern focused on the burden on patients of 
traveling to a larger hospital outside the island. In the US, the concern was both distance and a long waiting time for an appointment with a specialist.

(In urban area) you can do imaging and blood tests routinely to make a diagnosis but in the environment where we could not easily access testing, I was less comfortable in making a diagnosis of dementia when in a rural area. (Japan_Rural and Urban_17)

I'll usually send them out to the neuropsychiatric testing, and I've had people very willing to pay for that, although you wait a long, maybe six months, to get it scheduled and actually have it completed. (US_Urban_M03)

\section{Perceived Benefits of Dementia Diagnosis in Primary Care}

Participants in Japan and the US both described similar benefits of diagnosing dementia such as ensuring the safety of patients and the community, gaining access to additional care resources, making future plans, and improving the well-being of patients and their family members.

Many primary care physicians felt that diagnosing dementia was useful, first of all, to patients' family members to understand the cause of the demented person's problems in light of his or her environment. Physicians can then offer advice to patients' families on how to deal with problems associated with dementia symptoms of the patient. Diagnosing dementia was perceived as an opportunity for patients' families to re-evaluate their way of relating to their demented family members.

In some cases, I've noticed that patients' family members seemed relieved (to know the diagnosis). So we could then share problems in the presence of patients without feeling guilty. (Japan_Urban_04)

I think it's important...just helping the family understand that there's a medical reason for why maybe personality change is going on, help them understand the frustration, why a person can't do something anymore. (US_Rural_R10)

The formal establishment of the diagnosis of dementia was found beneficial for gaining access to appropriate care services and insurance eligibility. Primary care physicians in Japan and the US reported supporting their patients and their family members by discussing the prognosis by providing guidance on what will happen in the immediate future for the dementia patient, advising how to prepare, discussing the kinds of care appropriate for the patient. Planning places to live/die emerged as a major topic to be considered in the long-term.

If they can use long-term care services, there are various day service facilities. For instance, just to spend the daytime and come home, or those multi-functional type facilities that are very useful where the same staff provide home care and accept short-term stays in the facility. (Japan_Rural_23)

Patients having that diagnosis open the door up to other services that we can get, whether it be home health care, certain in-home devices, some medication administrative assistance. Even long-term care in extended-care facilities. (US_Rural_R11) 
Medications that are commonly used in Japan were donepezil, memantine, galantamine, and in the US were donepezil and memantine. However, many participants said that they did not see a clear effect of these medications for dementia. Even though both Japanese and the US participants explained to patients and their family members that medication could potentially slow down the progress of dementia, they did not impose medications on patients if they did not wish to take them.

There are those who want to take medication if available, and there are those who don't want to if the medications aren't so effective. It all depends, so I try to make sure to ask his/her preference at the beginning. (Japan_Urban_19)

I have very mixed feelings about even starting people on the medications because I feel like the evidence for them that they are effective is pretty limited. (US_Urban_U06)

There were participants from both countries who stated that they didn't see much value in specifying the type of dementia or making fine adjustments in dementia medications according to diagnoses. Such ideas were shared when contrasting their own practice to the typical approach of dementia specialists. As primary care physicians, they focused more on the mental care and life support for patients and care givers in the belief of pursuing holistic care by balancing the benefits of available treatment options.

\section{Diverse views on the timing of diagnosing dementia}

Primary care physicians had knowledge for establishing a dementia diagnosis and knew the benefits of diagnosing dementia. However, how and when to bring up the topic with patients could be a sensitive issue. Participants in Japan and the US shared similar recognition of features related to diagnosing dementia such as cognitive function usually declining slowly, that there could be diagnostic ambiguity, that there was social stigma associated with the disease, and a hesitancy to 'label' the patient.

In our probing about participant's memorable cases and their trajectories from the diagnosis to final treatment, the common triggers of suspecting dementia in patients were family members reporting symptoms and patients not taking medications. However, we observed different trends in the process of suspecting and determining dementia diagnosis in Japanese and the US participants. In Japan, primary care physicians described involvement of a wide range of individuals beyond the family members being involved in detecting dementia. For instance, receptionists or nurses may express concerns about patients based on their behavior outside examination rooms. There were reports of caseworkers or neighbors who were worried about patients' living conditions and sought help from clinics. Some Japanese physicians were building community networks by routinely attending meetings with agencies that managed security in the area such as fire and police departments.

I rarely had noticed the first signs of dementia. In the majority of cases, I understood for the first time after listening carefully to other people's opinions which suggested that patients possibly had dementia. (Japan_Rural_07) 
In many cases that the Japanese participants introduced to us, the relationship with a dementia patient often had started when there was suspicion that the patient had dementia. Another typical pattern occurred when a physician took over the responsibility of a patient's care towards the end of life, e.g., when the patient switched to home care and the patient was already diagnosed with dementia at the time.

Her dementia was already in advanced stage when I first saw her. She had been in a facility, but her daughter wanted to take care of this patient at home for the remaining time and asked me if I could provide home visits for them. (Japan_Urban_2)

In contrast, commonly, US primary care physicians reported having had a relationship with their patients from an earlier stage of their lives and were able to notice changes associated with dementia during wellness visits. It was observed that the process is often nuanced and slow to evolve. The fact that dementia screening was required in the annual wellness visits for patients who were covered on Medicare prompted physicians to be on the watch for early signs of cognitive decline.

I'm starting to get from actually our Medicare Annual Wellness Visit Form, which has a question that will say something about memory, and they'll circle, "Yes," just really not thinking anything of it. And then we start to have that conversation. (US_Urban_M04)

Only one physician in Japan reported screening for dementia as a regular part of a health check. In many cases Japanese physicians took a 'wait and see approach' until it became evident that patients' family members or neighbors were having problems. Under the long-term care insurance system in Japan, a practical benefit of diagnosing dementia was to meet eligibility criteria for services and to access benefits under the system. The long-term care insurance covers the involvement of both patient care coordinators called "care managers" and visiting nurses to support patients' lives including end-of-life care. The link between establishing the diagnosis of dementia and accessing long-term care services in practice was confirmed by reports of some physicians who viewed diagnosing dementia not as particularly meaningful on isolated islands where resources for public care services were limited and where family members and neighbors provided supportive care for the elderly.

I've been asked quite a few times to write documents that were required in order for patients to activate the long-term care services. (Japan_Urban and Rural_05)

The long-term care insurance does not offer that many services anyway, so I did not used to write that many letters indicating the diagnosis (of dementia on an island). (Japan_Rural, remote island_14)

Additionally, another trigger for US participants to make an early diagnosis was reported as coming from systematic prompting and incentives to have patients complete advance directives. In completing an advance directive, patients are able to legally name a surrogate decision-maker who could make decisions regarding medical procedures on behalf of patients who are no longer able to do it for themselves. Participants shared the belief that this was a way to secure a future consistent with the 
patient's preferences and that prompting for communication about this topic was built into the annual wellness visit.

We always want the patient to name someone who can make decisions for them. We do something like advance planning built into our screening process - all patients actually. (US_Urban_U01)

In contrast, Japanese primary care physicians often described the process of discussing housing and end-of-life care issues with families of patients with advanced dementia, but decision-making systems such as the advance directive were not mentioned as a motivator for early diagnoses of dementia.

Figure 1 illustrates the approach to diagnosing dementia by Japanese and the US primary care physicians and related factors found in this analysis. The diagram depicts a central arrow demarcating the progressive nature of a patient's dementia and cognitive decline from early stage until death. The bottom illustrates an overarching timeline of events for the trajectory in Japan and the top is an overarching timeline of events the trajectory in the US. In the diagram, "relationship" refers to the time of the physician being responsible for the patient's health care management. Many of the US primary care physicians had been seeing their dementia patients even before symptoms appeared, whereas participating Japanese physicians were more likely to have been consulted after family members identified concern about dementia. The social systems which frame the timing of diagnostic intervention of dementia were "long-term care insurance" in Japan and "advance directives" in the US. The concept of "advance care planning" was pervasive in the US. However, in Japan, advanced care planning was not raised as a motivation for early diagnosis but was sometimes mentioned in the context of end-of-life care.

\section{Discussion}

By comparing the experiences of 48 primary care physicians, 24 each in Japan and the US, we have identified similarities and differences in their perspectives about diagnosing dementia. Similarities were the clinical approaches for establishing a diagnosis of dementia, views on potential benefits to patients and family members of diagnosing dementia, and common values prioritizing the quality of life and wellbeing of patients and caregivers rather than a focus on the diagnosis or medical treatment of dementia. This reflects a widely advocated role for primary care doctors to provide patient-centered care in the context of long-term relationships with patients and their family members $[3,4,53]$.

The most striking differences between Japan and the US primary care physicians were perceptions about the appropriate timing and behaviors relative to diagnosing dementia. The US physicians were more proactive in diagnosing dementia in the early stages, while Japanese physicians tended to make the diagnosis when family members were encountering apparent problems. This difference seems to be influenced by their respective social systems available for supporting dementia patients. In the US, the primary care physicians described a system of dementia screening and promotion of advance directives [45] at regular health check-ups. In contrast, under the long-term care insurance system in Japan [35, 40], eligibility for services happens only when the need for care has emerged. 
Through this Japan-US comparison study, there are some points worth noting about both countries. The first thing that Japan could learn from the US is regular health check-ups done by primary care physicians could more thoroughly include active screening for dementia to promote early detection. Also, systematically incorporating and implementing an advance directive system could encourage people with dementia to prepare for the future while they are still capable of expressing their preferences for choosing a healthcare proxy. In Japan, screening for dementia is conducted as part of public care-prevention service by each local government $[31,54]$ or done when people request it. Most primary care physicians in this study were not regularly conducting such screening for their own patients. In addition, in the current Japanese adult guardianship system, a proxy can be appointed only for property management and not for medical decision-making [48]. Medical and nursing care plans for the elderly are often decided collectively by family members and medical and nursing professionals, considering what the patient would have wanted in that situation $[25,26,55]$. While efforts promoting advance care planning in Japan began in 2007 [47], uptake has been slow [56, 57]. But many agree that advance care planning as an approach enabling the elderly to prepare for the final stage of their lives by explicitly stating their preferences will gradually expand in the future $[47,56,58]$. When recognition that preferences expressed through advanced care planning are legally protected, medical professionals may feel more compelled to communicate with those suspected of having dementia at an earlier stage of the disease.

The diagnosis of dementia by primary care physicians in Japan was significantly linked to motivation to utilize long-term care services for patients and to support family caregivers. This indicates that use of the long-term care services for elderly care has become widely utilized since the enforcement of the LongTerm Care Insurance Act in 2000 in Japan [31, 59]. Under the insurance system, there are multiple care service providers in place that facilitate utilizing coordinated services under the medical diagnosis of dementia [32]. In this regard, we observed that in the US, patients' access to care support varied depending on the physician's practice environment, and a diagnosis of dementia was not always associated with enhanced care provision. This may be a challenge for dementia care in primary care in the US [3].

What can be inferred from the above discussion is that the concept of appropriate timing of diagnostic intervention for dementia reflects what medical or nursing care services physicians have available to them. The lack of testing and curative medication, and the social stigma associated with dementia serve as barriers to formally make the diagnosis and render disclosure difficult [26]. However, primary care physicians will intervene when they find benefits that outweigh these barriers. Therefore, not only the availability of early diagnostic measures such as biomarkers [60], but also the availability of systems and care services in place for patients that can be used after diagnosis is necessary to promote early diagnosis and advance care planning initiatives.

A strength of the present study is the collection of perspectives of primary care physicians about diagnosing dementia both in Japan and the US, and in rural and urban areas. By listening to various trajectories of long-term dementia care, we were also able to gain insights into sociocultural aspects of diagnosing dementia [20-22] and understanding physicians' intention behind their practices in depth. 
Moreover, by comparing the practices in two countries with different health care systems and cultural backgrounds, with a multicultural research team, we were able to explore the characteristics of both countries that may not be easily recognized by examining one system in isolation [20,23]. Comparative studies of countries such as this would be useful in examining different methods and systems beyond existing systematic boundaries [27].

A potential limitation, or at least a particular challenge of cross-national research, especially in a qualitative study, involves the selection of comparable participants. In the present study, participants were predominantly recruited from the membership of the Japan Primary Care Association while in the case of the US, they were all primary care physicians in the state of Michigan. We do feel the findings are transferrable in both Japan, and for the Michigan sample to the broader US. These findings identify the kinds of challenges and approaches characteristic in each country. It is plausible that the interviewers' differences in backgrounds could have impacted the information drawn from the participants in the two countries since the interviewer is the instrument of data collection [49]. Still, in spite of geographic, language, and sampling strategy challenges, we believe a rigorous approach was followed.

\section{Conclusion}

The challenges faced and strategies used by Japan and US primary care physicians are remarkably similar relative to diagnosing dementia. That said, there are differences. The US primary care physicians were more proactive in diagnosing dementia in the early stages, while Japanese counterparts had a tendency to wait until the problems faced by the family members became apparent. Efforts promoting the early diagnosis of dementia need consider not only the testing measures available, but also the longterm care services and an advance directive system that makes establishing diagnosis clearly beneficial to patients.

\section{Abbreviations}

US: United States

GRIN: Great Lakes Research Into Practice Network

MMSE: Mini-Mental State Examination

HSD-R: Hasegawa's Dementia Scale

MoCA: Montreal Cognitive Assessment

IRB: Institutional Review Board

\section{Declarations}

\section{Ethics approval and consent to participate}


Informed consent was obtained from all human study participants and ethical approval was obtained from the Institutional Review Board of Hamamatsu University School of Medicine in Japan (No.16-233) and Health Sciences and Behavioral Sciences Institutional Review Board of the University of Michigan in the US (IRB 00000246). All methods were performed in accordance with the relevant guidelines and regulations set by the IRB.

\section{Consent for publication}

Not applicable.

\section{Availability of data and materials}

The datasets used and/or analyzed during the current study are available from the corresponding author on reasonable request.

\section{Competing interests}

The authors declare that they have no competing interests.

\section{Funding}

This research was funded as an International Collaborative Research Project by Pfizer Health Research Foundation from 2016 to 2018 and Hamamatsu University School of Medicine Grant-in-Aid in 2018. The conception and design of the original US research received pilot funding from the Michigan Center on the Demography of Aging (NIA P30AG012846), "Rural Older Adults with Cognitive Impairment and Chronic Disease: A Mixed Methods Study of Social and Health Factors in Their Care". The funding bodies had no role in the design of the study, including collection, analysis, and interpretation of data, or in writing the manuscript.

\section{Authors' contributions}

The individual contribution of the authors are: MF, CC and ST designed the research, MA, MD, ER, and KP collected and coded data and CC, ST, MM were involved in analyzing the data. MF and MI managed the research project, provided supervision, and revised the draft critically for important intellectual content. All authors read and approved the final manuscript.

\section{Acknowledgments}

The authors thank all the primary care physicians who participated in this investigation. In addition, we would like to thank Dr. Sarah Pettibone, a medical and public health student at the time of the study, for helping to conduct interviews in Michigan. Satoko Motohara and Rania Ajilat kindly assisted with the member checking process in Michigan, and the latter with manuscript preparation.

\section{References}


1. Livingston G, Sommerlad A, Orgeta V, Costafreda SG, Huntley J, Ames D, et al. Dementia prevention, intervention, and care. Lancet. 2017;390(10113):2673-734.

2. Boustani M. Dementia screening in primary care: not too fast! J Am Geriatr Soc. 2013;61(7):1205-7.

3. Galvin JE, Sadowsky CH. Practical guidelines for the recognition and diagnosis of dementia. J Am Board Fam Med. 2012;25(3):367-82.

4. Iliffe S, Manthorpe J, Eden A. Sooner or later? Issues in the early diagnosis of dementia in general practice: a qualitative study. Fam Pract. 2003;20(4):376-81.

5. Patnode CD, Perdue LA, Rossom RC, Rushkin MC, Redmond N, Thomas RG, et al. Screening for cognitive impairment in older adults: an evidence update for the U.S. Preventive Services Task Force. Evidence Synthesis No. 189. Rockville, MD: Agency for Healthcare Research and Quality; 2020.

6. Smith T, Cross J, Poland F, Clay F, Brookes A, Maidment I, et al. Systematic review investigating multidisciplinary team approaches to screening and early diagnosis of dementia in primary care - what are the positive and negative effects and who should deliver it? Curr Alzheimer Res. 2018;15(1):5-17.

7. Le Couteur DG, Brayne $C$. Should family physicians routinely screen patients for cognitive impairment? No: screening has been inappropriately urged despite absence of evidence. Am Fam Physician. 2014;89(11):864-5.

8. Health and Global Policy Institute, McCann Global Health. Social prescription for dementia (in Japanese). Tokyo: Health and Global Policy Institute; 2017. Available from: https://hgpi.org/research/747.html.

9. Uragami k. The use of dementia treatment guidelines in daily clinical practice. The Japanese Society of Internal Medicine. 2008;97(12):180-5.

10. Editorial Committee of Clinical Practice Guideline for Dementia. Systems and social resources to support people with dementia and their families (in Japanese). In: Clinical Practice Guideline for Dementia [Internet]. Tokyo: Igaku-Shoin; 2017. p. 170-201. https://www.neurologyjp.org/guidelinem/degl/degl_2017_05.pdf.

11. McCarten JR, Borson S. Should family physicians routinely screen patients for cognitive impairment? Yes: screening is the first step toward improving care. Am Fam Physician. 2014;89(11):861-2.

12. Boise L, Camicioli R, Morgan DL, Rose JH, Congleton L. Diagnosing dementia: perspectives of primary care physicians. Gerontologist. 1999;39(4):457-64.

13. Porteri C. Advance directives as a tool to respect patients' values and preferences: discussion on the case of Alzheimer's disease. BMC Med Ethics. 2018;19(1):9.

14. Burlá C, Rego G, Nunes R. Alzheimer, dementia and the living will: a proposal. Med Health Care Philos. 2014;17(3):389-95.

15. Moyer VA, U.S. Preventive Services Task Force. Screening for cognitive impairment in older adults: U.S. Preventive Services Task Force recommendation statement. Ann Intern Med. 2014;160(11):7917. 
16. Iliffe S, Koch T, Jain P, Lefford F, Wong G, Warner A, et al. Developing an educational intervention on dementia diagnosis and management in primary care for the EVIDEM-ED trial. Trials. 2012;13:142.

17. Lathren CR, Sloane PD, Hoyle JD, Zimmerman S, Kaufer DI. Improving dementia diagnosis and management in primary care: a cohort study of the impact of a training and support program on physician competency, practice patterns, and community linkages. BMC Geriatr. 2013;13:134.

18. Noda H, Yamagishi K, Ikeda A, Asada T, Iso H. Identification of dementia using standard clinical assessments by primary care physicians in Japan. Geriatr Gerontol Int. 2018;18(5):738-44.

19. Cabinet Office Government of Japan. 2017 white paper on aging society (in Japanese). Tokyo: Cabinet Office; 2017. https://www8.cao.go.jp/kourei/whitepaper/w-2017/zenbun/pdf/1s1s_01.pdf.

20. Hillman A, Latimer J. Cultural representations of dementia. PLoS Med. 2017;14(3):e1002274.

21. Cipriani G, Borin G. Understanding dementia in the sociocultural context: a review. Int J Soc Psychiatry. 2015;61(2):198-204.

22. Henderson JN, Traphagan JW. Cultural factors in dementia: perspectives from the anthropology of aging. Alzheimer Dis Assoc Disord. 2005;19(4):272-4.

23. Brayne C, Miller B. Dementia and aging populations-A global priority for contextualized research and health policy. PLoS Med. 2017;14(3):e1002275.

24. Nakanishi M, Nakashima T. Features of the Japanese national dementia strategy in comparison with international dementia policies: how should a national dementia policy interact with the public health- and social-care systems? Alzheimers Dement. 2014;10(4):468-76.e3.

25. Fetters MD, Danis M. We live too short, and die too long - Japanese and U.S. physicians' caregiving practices and approaches to withholding life-sustaining treatments. In: Long SO, editor. Caring for the elderly in Japan and the U.S. London: Routledge; 2000. p. 122-45.

26. Abe M, Tsunawaki S, Matsuda M, Cigolle CT, Fetters MD, Inoue M. Perspectives on disclosure of the dementia diagnosis among primary care physicians in Japan: a qualitatively driven mixed methods study. BMC Fam Pract. 2019;20(1):69.

27. Lindsay S. Five approaches to qualitative comparison groups in health research: a scoping review. Qual Health Res. 2019;29(3):455-68.

28. OECD. Share of the population aged over 65 and 80 years, 2017 and 2050. In: Health at a glance 2019: OECD indicators. Paris: OECD Publishing; 2019. p. 219. Accessed 13 Aug 2020.

https://www.oecd-ilibrary.org/docserver/4dd50c09-en.pdf?

expires=1611863747\&id=id\&accname=guest\&checksum=479D69B7064A80E04D48E36025B378CC

29. OECD. Elderly population (indicator). https://data.oecd.org/pop/elderly-population.htm\#indicatorchart. Accessed 13 Aug 2020.

30. United States Census Bureau. Quick Facts (population). https://www.census.gov/quickfacts/fact/table/MI/PST045219. Accessed 13 Aug 2020.

31. Health and Welfare Bureau for the Elderly, Ministry of Health Labour and Welfare. Long-term care insurance system of Japan. https://www.mhlw.go.jp/english/policy/care-welfare/care-welfare- 
elderly/dl/ltcisj_e.pdf. 2016. Accessed 13 Aug 2020.

32. Ministry of Health Labour and Welfare. Comprehensive services/preventive care and support for daily living (in Japanese). https://www.mhlw.go.jp/stf/seisakunitsuite/bunya/0000192992.html. Accessed 13 Aug 2020.

33. Alzheimer's Association. 2020 Alzheimer's disease facts and figures. Alzheimers Dement. 2020. doi: 10.1002/alz.12068

34. Ikegami N, Yoo BK, Hashimoto H, Matsumoto M, Ogata H, Babazono A, et al. Japanese universal health coverage: evolution, achievements, and challenges. Lancet. 2011;378(9796):1106-15.

35. Ikegami N. Financing long-term care: lessons from Japan. Int J Health Policy Manag. 2019;8(8):4626.

36. Ishibashi M. The U.S. health care insurance system - Introduction of a universal health care system and attempts to control medical costs by using private insurance companies (in Japanese). Tokyo: Daiwa Institute of Research; 2013.

37. LaPonsie M. The high cost of long-term care insurance (and what to use instead). U.S. News. 2019. https://money.usnews.com/money/personal-finance/family-finance/articles/the-high-cost-of-longterm-care-insurance-and-what-to-use-instead. Accessed 13 Aug 2020.

38. Kato D, Ryu H, Matsumoto T, Abe K, Kaneko M, Ko M, et al. Building primary care in Japan: Literature review. J Gen Fam Med. 2019;20(5):170-9.

39. Friedberg MW, Hussey PS, Schneider EC. Primary care: a critical review of the evidence on quality and costs of health care. Health Affairs. 2010;29(5):766-72.

40. Hatano Y, Matsumoto M, Okita M, Inoue K, Takeuchi K, Tsutsui T, et al. The vanguard of communitybased integrated care in Japan: the effect of a rural town on national policy. Int $\mathrm{J}$ Integr Care. 2017;17(2):2.

41. Zazove $P$, Inoue M, Motohara S, Fetters MD. Benefits of having departments of family medicine in universities. J Gen Fam Med. 2015;38(4):358-68.

42. Saigal P, Takemura Y, Nishiue T, Fetters MD. Factors considered by medical students when formulating their specialty preferences in Japan: findings from a qualitative study. BMC Medical Education. 2007;7(1):31.

43. Fetters MD, Yokoyama I. Medical education in Japan. In: The SAGE handbook of modern Japanese studies. Newbury Park, CA: SAGE; 2015. p. 314.

44. Ruhnke GW, Wilson SR, Akamatsu T, Kinoue T, Takashima Y, Goldstein MK, et al. Ethical decision making and patient autonomy. Chest. 2000;118(4):1172-82.

45. Brown BA. The history of advance directives. A literature review. J Gerontol Nurs. 2003;29(9):4-14.

46. Dresser R. Medicare and advance planning: the importance of context. Hastings Center Report. 2016;46(3):5-6.

47. Inoue M, Hanari K, Hamano J, Gallagher J, Tamiya N. Current engagement in advance care planning in Japan and its associated factors. Gerontol Geriatr Med. 2019;5:2333721419892694. 
48. Narumoto J. Expectations for psychology in decision-making support for people with dementia (in Japanese). Academic Trends. 2019;24(5):32-6.

49. Dejonckheere M, Vaughn LM. Semistructured interviewing in primary care research: a balance of relationship and rigour. FMCH. 2019;7(2):e000057.

50. Agency for Healthcare Research and Quality. Great lakes research into practice network. https://pbrn.ahrq.gov/pbrn-registry/great-lakes-research-practice-network. Accessed 13 Aug 2020.

51. Fetters MD, Rubinstein EB. The $3 \mathrm{Cs}$ of content, context, and concepts: a practical approach to recording unstructured field observations. Ann Fam Med. 2019;17(6):554-60.

52. Creswell JW, Miller DL. Determining validity in qualitative inquiry. Theory into Practice. 2000;39(3):124-30.

53. Tilburgs B, Vernooij-Dassen M, Koopmans R, van Gennip H, Engels Y, Perry M. Barriers and facilitators for GPs in dementia advance care planning: a systematic integrative review. Plos One. 2018;13(6).

54. LIFUL. What it the general services for preventing long-term care and supporting daily living (in Japanese). https://kaigo.homes.co.jp/manual/homecare/zaitaku_service/sogo/. Accessed 13 Aug 2020.

55. Hatano Y, Fukui K. The problem with informed consent and the role of psychiatrists in an aging society. Jpn J Gen Hosp Psychiatry 2013;25(4).

56. Shimada C, Hirayama R, Wakui T, Nakazato K, Obuchi S, Ishizaki T, et al. Reconsidering long-term care in the end-of-life context in Japan. Geriatr Gerontol Int. 2016;16:132-9.

57. Fetters MD. Bioethics and medico-legal issues in Japan. In: Sage handbook of modern Japanese studies. Newbury Park: SAGE; 2015. p. 325.

58. Aoki T, Miyashita J, Yamamoto Y, Ikenoue T, Kise M, Fujinuma Y, et al. Patient experience of primary care and advance care planning: a multicentre cross-sectional study in Japan. Fam Pract. 2017;34(2):206-12.

59. Japanese Law Translation. Long-term care insurance act 1997. http://www.japaneselawtranslation.go.jp/law/detail/?id=94\&vm=\&re=. Accessed 13 Aug 2020.

60. Vanderschaeghe G, Dierickx K, Vandenberghe R. Review of the ethical issues of a biomarker-based diagnoses in the early stage of alzheimer's disease. J Bioeth Inq. 2018;15(2):219-30.

\section{Figures}




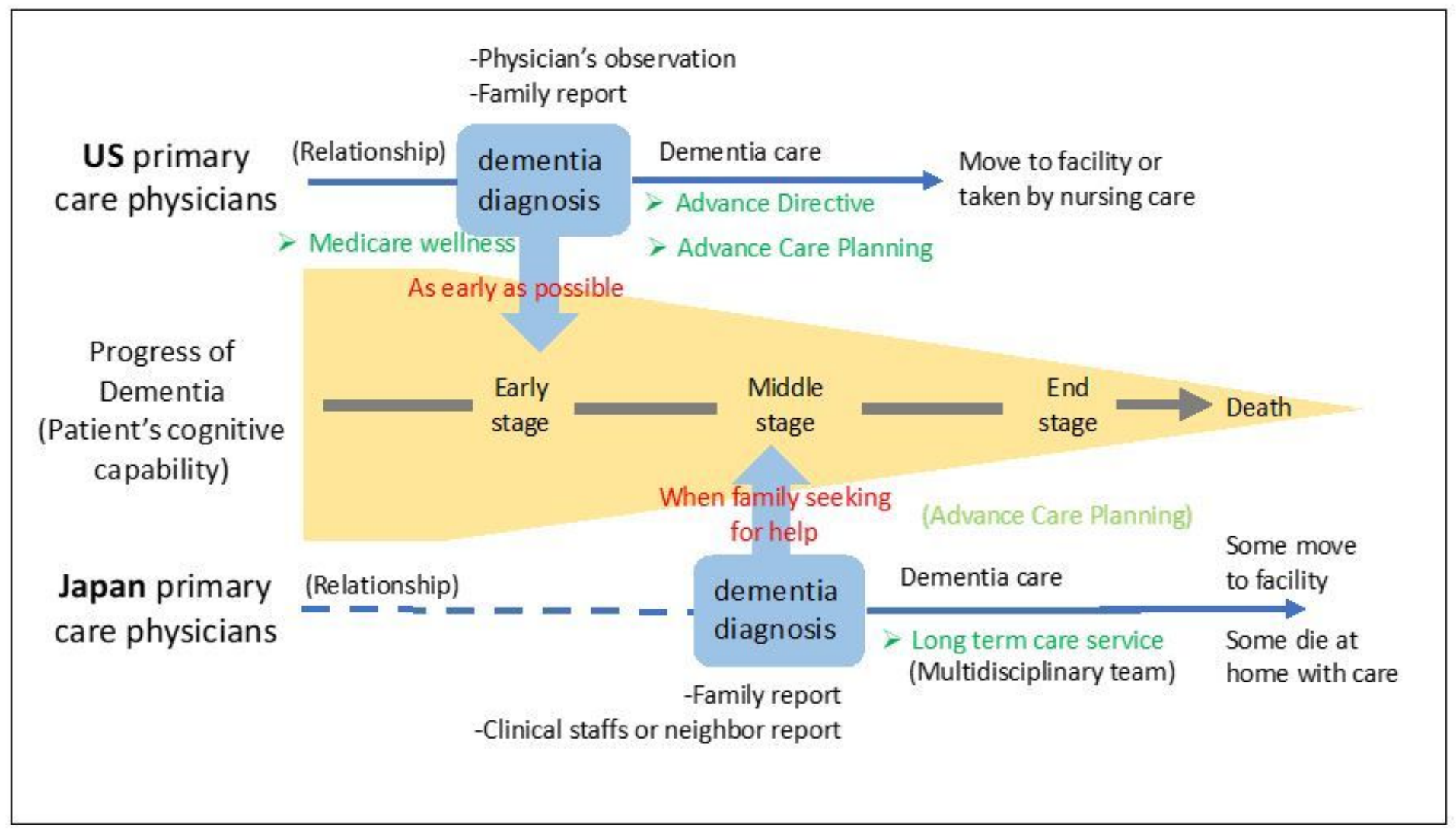

\section{Figure 1}

Primary care physicians' approaches to diagnosing dementia and factors affected:

\section{Supplementary Files}

This is a list of supplementary files associated with this preprint. Click to download.

- Supplement1.interviewguide1.docx 